\title{
Survey of Anxiety and Depression in Patients with Suspected and Confirmed Cases of COVID-19 During Hospitalization and Isolation
}

\section{Meifang Mai}

Fifth Affiliated Hospital of Sun Yat-sen University

Juan Wang ( $\nabla$ wangjuanzhang@163.com )

Fifth Affiliated Hospital of Sun Yat-sen University

Dongliang Xia ( $\nabla$ xd11000@sina.com.cn )

Fifth Affiliated Hospital of Sun Yat-sen University

\section{Xinming Guo}

Fifth Affiliated Hospital of Sun Yat-sen University

Fei Li

Fifth Affiliated Hospital of Sun Yat-sen University

\section{Yilan Chen}

Fifth Affiliated Hospital of Sun Yat-sen University

\section{Xiaohua Gong}

Fifth Affiliated Hospital of Sun Yat-sen University

\section{Zhonghe Li}

Fifth Affiliated Hospital of Sun Yat-sen University

Huitao Zhang ( $\square$ huitao-198@163.com)

Fifth Affiliated Hospital of Sun Yat-sen University https://orcid.org/0000-0002-6171-9604

\section{Research Article}

Keywords: COVID-19, anxiety, depression, sleep disorder, hospitalization, isolation

Posted Date: April 8th, 2020

DOI: https://doi.org/10.21203/rs.3.rs-17959/v1

License: (c) (i) This work is licensed under a Creative Commons Attribution 4.0 International License. Read Full License 


\section{Abstract}

Objective: To observe the anxiety and depression experienced by patients with suspected and confirmed COVID-19 during hospitalization and isolation.

Methods: A cross-sectional survey was performed with 66 patients with suspected and confirmed COVID19 who were admitted to the Fifth Affiliated Hospital of Sun Yat-sen University from February 9, 2020, to February 22, 2020. The data collected including sex, age, education level, and nucleic acid test results. Anxiety, depression, and sleep disorders were evaluated using the Self-Rating Anxiety Scale (SAS), SelfRating Depression Scale (SDS) and Pittsburgh Sleep Quality Index Scale (PSQI), respectively.

Statistical analysis: SPSS 23.0 software was used for data processing, and multifactor logistic regression analysis was used to identify the independent risk factors. Spearman correlation analysis was used to study the correlations among the observed indicators; $P<0.05$ was considered statistically significant.

Results: The incidences of anxiety, depression, and sleep disorders in the suspected case group were $18.2 \%, 18.2 \%$, and $39.4 \%$; the incidences of anxiety, depression, and sleep disorders in the diagnosed group were $42.4 \%, 57.6 \%$, and $69.7 \%$. The incidence of sleep disorders was higher than the domestic norm, and the difference was statistically significant $(P<0.05)$. Logistic regression analysis adjusted for multiple factors showed that the main factor affecting anxiety was age; the main factors affecting depression were age and a positive nucleic acid test; and the main factor affecting sleep disorders was age. The anxiety, depression and sleep disorder scores were significantly positively correlated $(P<0.05)$.

Conclusion: Patients with suspected and confirmed COVID-19, especially people aged 50 years and over, have significant anxiety, depression and sleep disorders. Anxiety, depression and sleep disorders in patients with confirmed cases are more serious than those in patients with suspected cases. It is necessary to focus on the psychological state of such patients, actively conduct psychological counseling, and reduce their anxiety and depression.

\section{Background}

In December 2019, Wuhan City, Hubei Province, China, was the site of multiple cases of pneumonia related to infection with a novel coronavirus [1]. With the spread of the epidemic, such cases have been found in other parts of China and abroad. As an acute respiratory infectious disease, the disease has been included as one of the Class $B$ infectious diseases stipulated in the Law of the People's Republic of China on the Prevention and Control of Infectious Diseases and is currently being managed as a Class A infectious disease. On February 11, 2020, the WHO officially named the new illness coronavirus disease 2019 (COVID-19) [2]. The disease has a long incubation period and a high infection rate, and those infected with the virus can become sources of infection [3-6], with a wide range of transmission. At present, no clinically effective drugs have been found. Therefore, the state stipulates that suspected and confirmed cases should be isolated and treated in designated hospitals with effective isolation and 
protection conditions; suspected cases must be treated in a single room and isolated, although multiple confirmed cases can be treated in the same ward. Isolation during treatment, the strict prevention and control requirements, and patients' lack of communication with the outside world render the patients prone to mental and psychological problems such as anxiety and depression. Furthermore, the negative emotions involved in anxiety and depression can lead to sleep disorders, decreased physical immunity, and various organ dysfunctions. A reduced physical performance status is not conducive to recovery and rehabilitation. The National Health Council issued guidelines for emergency psychological crisis interventions for people affected by COVID-19 [7]. This study investigates the status of anxiety, depression, and sleep disorders in patients with suspected and confirmed COVID-19, with a view to providing a reference for the identification of anxiety and depression among patients with suspected and confirmed COVID-19.

\section{Survey Objects And Methods}

\section{Research object}

Sixty-six patients with suspected and confirmed COVID-19 who were admitted to the Fifth Affiliated Hospital of Sun Yat-sen University from February 9, 2020, to February 22, 2020 were selected as the research subjects. The inclusion criteria were as follows: (1) patients with suspected or confirmed cases of COVID-19 based on the diagnostic criteria in the "New Coronavirus Pneumonia Diagnosis and Treatment Case (6th Edition)" who were admitted to the COVID-19 area of the hospital, [8]; (2) age $\geq 18$ years; and (3) voluntary participation in the study and ability to cooperate with the study procedures. The exclusion criteria were as follows: (1) severe mental disorders; (2) language communication barriers; (3) inability to answer the survey questions or communicate normally.

\section{Survey Tools}

The researchers designed a questionnaire to collect general information and disease-related data from the research subjects. The general information included the patient's name, sex, age, marital status, education level, etc. The disease-related information included the course of the disease and nucleic acid test results.

The Self-Rating Depression Scale (SDS) was developed by Zung (Practical Manual of Nursing Management Tools and Methods) and is used to assess the frequency of symptoms in the past week. The scale includes 20 items. Each item is scored on a scale from 1 to 4 . The scores for the 20 items are added together to obtain a rough score, which ranges from 20 to 80 points. A rough score greater than 40 points suggests depression. The higher the score, the more severe the degree of depression is. The rough score is then multiplied score by 1.25 and rounded to the nearest whole number to obtain the standard score. A standard score greater than 50 indicates the presence of depression. [Note: The scale collaboration group evaluated 1,340 Chinese people and found a rough score of $33.46 \pm 8.55$ points and a standard score of $41.88 \pm 10.57$ points; these scores were used as the domestic norms]. 
The Self-Rating Anxiety Scale (SAS) was developed by Zung and is used to assess the frequency of occurrence of various symptoms in the past week. The scale includes 20 items, each of which is scored on a scale from 1 to 4 . The scores for the 20 items are added together to obtain a rough score. The rough score ranges from 20 to 80 points. A rough score $>40$ points indicates anxiety. The higher the score, the greater the severity of anxiety is. Multiplying the rough score by 1.25 and rounding to the nearest whole number provides the standard score. Anxiety is considered present when the standard score is above 50. (Note: The scale collaboration group evaluated 1158 Chinese individuals and found a rough score of $29 \pm$ 10.07 points, which was used as the domestic norm).

The Pittsburgh Sleep Quality Index (PSQI) consists of 9 self-assessed questions and 5 other items, including 7 factors. Each factor is scored on a scale from 0 to 3 , and the total sleep quality score is calculated based on those factors. The range is 0 to 21 points, and the higher the score is, the worse the sleep quality. A total score> 7 points indicates the existence of sleep problems.

\section{Data collection method}

The surveys were administered face-to-face. A doctor and a nurse in the COVID-19 area of the hospital selected patients with suspected and confirmed COVID-19 according to the inclusion and exclusion criteria. The questionnaires were completed and returned on the spot. A total of 69 questionnaires were issued, and 66 valid questionnaires were retrieved. The recovery rate was $95.7 \%$.

\section{Statistical processing}

Data processing was performed using SPSS 23.0 software. Normally distributed measurement data are expressed as the mean \pm standard deviation, while nonnormally distributed measurement data are expressed as the median (interquartile range). Count data are expressed as cases (percentage), and comparisons between groups were performed using the $\chi^{2}$ test. Multivariate logistic regression analysis was used to identify the independent risk factors. Spearman correlation analysis was used to study the correlations among the observed indicators. $P<0.05$ was considered statistically significant.

\section{Results}

\section{Comparison of general information of study objects}

A total of 66 patients were included in the study, all of whom were from the Fifth Affiliated Hospital of Sun Yat-sen University, including 33 males and 33 females. The age range was from 19 to 72 years, with an average age of 39 years. In total, 26 people had a junior high school education or below, while 40 people had a high school education and above. With regard to the nucleic acid test results, 33 patients were positive, and 33 patients were negative. Of the included patients, 52 were married, and 14 were unmarried. (See Table 1 for details)

Table 1 General information of all patients 


\begin{tabular}{|c|c|}
\hline Sex (male/female, $\mathrm{n}$ ) & $33 / 33$ \\
\hline Age [years, median (quantiles)] & 39.0 (35.0 to 56.3 ) \\
\hline Married [ n (\%) ] & $52(78.8)$ \\
\hline Education [ n (\%) ] & \\
\hline Junior school and below & $26(39.4)$ \\
\hline Senior school and above & $40(60.6)$ \\
\hline Hospitalized days [n, median (quantiles)] & $7.0(2.8$ to 11.5$)$ \\
\hline Viral nucleic acid test positive [ $\mathrm{n}(\%)]$ & $33(50.0)$ \\
\hline Anxiety score [n, median (quantiles)] & 41.3 (37.2 to 53.8 ) \\
\hline Depression score $[\mathrm{n}$, median (quantiles)] & 47.5 (40.0 to 58.8$)$ \\
\hline Sleep dysfunction score ( $\mathrm{n}$, mean \pm standard deviation) & $7.6 \pm 4.7$ \\
\hline
\end{tabular}

\section{Relationships between sex, age, education level, and nucleic acid test results and anxiety, depression, and sleep disorders}

The incidences of anxiety, depression, and sleep disorders in patients with suspected and confirmed cases of COVID-19 were not significantly different based on sex, marital status, and education level. The proportions of patients with anxiety, depression, and sleep disorders were higher among patients older than 50 years of age and among patients with positive nucleic acid tests. The differences were statistically significant. (See Table 2 for details)

Table 2 Relationships of sex, age, education and viral nucleic acid test results with degrees of anxiety, depression and sleep dysfunction $[\mathrm{n}(\%)]$ 


\begin{tabular}{|c|c|c|c|}
\hline Item & Anxiety & Depression & Sleep dysfunction \\
\hline \multicolumn{4}{|l|}{ Sex } \\
\hline Male & $12(36.4)$ & $13(39.4)$ & 16 (48.5) \\
\hline Female & $8(24.2)$ & $12(36.4)$ & $20(60.6)$ \\
\hline \multicolumn{4}{|l|}{ Age (years) } \\
\hline$<50$ & $7(16.7) *$ & $11(26.2)^{*}$ & $16(38.1)^{*}$ \\
\hline$\geqq 50$ & $13(54.2)^{*}$ & $14(58.3)^{*}$ & $20(83.3)^{*}$ \\
\hline \multicolumn{4}{|l|}{ Married } \\
\hline No & $5(35.7)$ & $6(42.9)$ & $5(35.7)$ \\
\hline Yes & $15(28.8)$ & 19 (36.5) & 31 (59.6) \\
\hline \multicolumn{4}{|l|}{ Education } \\
\hline Junior school and below & $8(30.8)$ & $10(38.5)$ & $14(53.8)$ \\
\hline Senior school and above & $12(30.0)$ & $15(37.5)$ & $22(55.0)$ \\
\hline \multicolumn{4}{|l|}{ Viral nucleic acid test } \\
\hline Negative & $6(18.2)^{*}$ & $6(18.2)^{*}$ & $13(39.4)^{*}$ \\
\hline Positive & $14(42.4)^{*}$ & 19 (57.6)* & $23(69.7)^{*}$ \\
\hline
\end{tabular}

* $P<0.05$

\section{Logistic regression analysis of risk factors for anxiety and depression symptoms}

Logistic regression models were established with and without anxiety, depression, and sleep disorders as the dependent variables. After analysis of multiple factors, the results showed that age influenced anxiety and depression in patients with suspected and confirmed COVID-19, while age and a positive nucleic acid test affected sleep disorders. (See Table 3 for details)

Table 3 Logistic regression analysis of risk factors for anxiety, depression and sleep dysfunction

\begin{tabular}{lccccc}
\hline \multicolumn{1}{c}{ Factor } & $B$ & $S$ & Wald & $P$ value & OR (95\% CI) \\
\hline $\begin{array}{l}\text { Anxiety } \\
\text { Age }\end{array}$ & 0.09 & 0.03 & 8.84 & $<0.01$ & 1.09 (1.03 to 1.15) \\
$\begin{array}{l}\text { Depression } \\
\text { Age }\end{array}$ & 0.05 & 0.03 & 4.27 & 0.04 & $1.06(1.00$ to 1.11$)$ \\
Viral nucleic acid test & 2.12 & 0.70 & 9.08 & $<0.01$ & $8.29(2.09$ to 32.81) \\
Sleep dysfunction & & & & & \\
Age & 0.09 & 0.03 & 8.74 & $<0.01$ & $1.09(1.03$ to 1.15$)$ \\
\hline
\end{tabular}

Adjusted sex, age, marital status, education, hospitalized days and viral nucleic acid test for all the models. 


\section{Correlations among anxiety, depression, and sleep disorder scores}

There were significant positive correlations between sleep disorders and anxiety and depression; that is, the more severe the anxiety or depression, the more likely the patients were to have sleep disorders. (See Figure 1, Figure 2, Figure 3)

\section{Discussion}

COVID-19 is a new, highly infectious disease with varying incubation periods and clinical manifestations; elderly patients and those with chronic diseases appear to be at the greatest risk. It has substantially affected normal life, work and study. In late January 2020, China initiated prevention and control measures for COVID-19 and initiated the "first-level emergency response". COVID-19 has become a severe epidemic that constitutes a major public health emergency, and it is spreading rapidly. People are prone to various psychological problems during the epidemic.

Patients with suspected and confirmed cases of COVID-19 require strict medical observation and isolation during treatment. During this period, during which they lack face-to-face communication with people, they often experience serious concerns, fear, depression and anxiety; in addition, the patients must endure coughing, fever, dyspnea and other physical discomfort, which all have effects on their psychological state.

On January 17, 2020, our hospital started to treat the first cases of COVID-19 in Zhuhai. Currently, 98 patients have been diagnosed, and more than 200 patients with suspected cases of COVID-19 have been admitted. During the treatment of COVID-19, we often see young mothers who are anxious about whether their children will be well taken care of at home. Even when patients experience improvements in their symptoms, they still worry about fluctuations in their condition. Some patients' body temperature may increase slightly, and if there is an increase, the patients become nervous and anxious. Some patients suffer from poor sleep due to anxiety, physical dysfunction, and symptoms such as chest tightness, shortness of breath, fatigue and poor appetite, which are not conducive to the treatment of and recovery from the disease.

This study conducted a cross-sectional survey of 66 patients with suspected and confirmed cases of COVID-19 during hospitalization and isolation and found that psychological problems such as anxiety, depression, and sleep disorders were common in all patients. The patients had a mean anxiety score of 41.3 points, a mean depression score of 47.5 points, and a mean sleep disorder score of $7.6 \pm 4.7$ points. The incidences of anxiety, depression, and sleep disorders were higher than domestic norms among these patients, and the differences were statistically significant $(P<0.05)$; this finding indicates that these patients experienced anxiety and depression. In addition to the abovementioned common factors, anxiety and depression can result from isolation and the general concern about the progress of the epidemic in the population. Most of the medical staff's energy is focused on treating the physical symptoms of the 
infection, which reduces their ability to intervene in psychological conditions. With the current shortage of protective materials and personnel, it is difficult to conduct routine psychological consultations and provide treatment [9]. This is consistent with the results of Cheng Li's research [10], which showed that patients with COVID-19 had significant anxiety; during the SARS epidemic in 2003, there were also studies that showed that patients with suspected cases of SARS who were totally isolated experienced anxiety, fear, loneliness and other psychological problems [11]. During the Ebola outbreak in Sierra Leone from 2014 to 2015, the number of people with mental illnesses in the region increased significantly: 71 patients (50\%) experienced mild pain, depression, anxiety, grief or social problems, while thirty patients developed psychosis requiring medication [12]. Therefore, during epidemics of infectious diseases, monitoring patients' psychological behavior and providing interventions at an early stage is very important for their rehabilitation.

Currently, most patients with COVID-19 have a good prognosis, while a few patients are critically ill. Elderly patients and those with chronic underlying diseases have a poor prognosis, while the symptoms are relatively mild in children. The investigation found that the incidences of anxiety, depression, and sleep disorders were relatively higher in confirmed cases; the survey results showed that in patients with a negative nucleic acid test, the incidence of anxiety was $18.2 \%$, and the incidence of sleep disorders was $39.4 \%$. Among patients with a positive nucleic acid test, the incidence of anxiety was $42.41 \%$, the incidence of depression was $57.6 \%$, and the incidence of sleep disorders was $69.7 \%$. These incidences were much higher than those in patients with a negative nucleic acid test, and the differences were statistically significant. These differences are related to the relatively severe physical symptoms in the diagnosed patients, who may worry that the disease will be difficult to cure or that it will affect their future work and burden their family. At the time of the SARS outbreak, Gabriel M. Leung found that those who were aware of their higher likelihood of infection and those who ultimately died of SARS had significantly higher anxiety scores than their counterparts. The level of anxiety is closely related to the intensity of the outbreak and to the number of new cases per day [13]. In this study, Spearman correlation analysis was used to analyze the relationships among the anxiety, depression, and sleep disorder scores. There were significant positive correlations among anxiety, depression, and sleep disorders. There are currently no specific therapeutic drugs for COVID-19. The main treatment methods are systemic supportive therapy and improving the patient's own immunity. Sleep disorders can lead to a decline in the body's immunity, adversely affecting the host's resistance to infectious diseases, and aggravating the severity of the disease [14-16]. Timely counseling in response to the psychological problems experienced by patients can alleviate negative emotions and assist in treatment in ways that cannot be achieved by drugs [11]; therefore, during the treatment of COVID-19, medical staff should pay attention to the impact of psychological disorders on the disease. The timely identification of problems and the initiation of clinical interventions, if necessary, may better help patients overcome these difficulties.

Gabriel M. Leung also found that women, people between the ages of 30 and 49, and people with a primary education or below were more likely to feel anxious [13]. However, the results of this study show that sociological factors such as education and sex had no significant effect on anxiety and depression in patients with COVID-19. This may be related to China's national mobilization, consistent epidemic 
prevention measures, and strong communication strategies by the news media. Most patients have a strong awareness of the disease, and the country's free treatment of COVID-19 reduces the economic pressure on patients. The proportions of patients with anxiety, depression, and sleep disorders were high, and the differences in incidence between patients and the general population norms were statistically significant. Considering that the elderly population is more likely to have chronic diseases and that patients with underlying diseases have a worse prognosis, these patients are experiencing physical discomfort and are concerned about their own condition progressing at any time. Symptoms of anxiety and depression are aggravated, which induces or aggravates sleep disorders. Sleep disorders can reduce the body's resistance to disease. COVID-19 is more likely to become severe in the elderly population, and the prognosis is poor [2, 4]; this prompts our medical staff to pay attention to the diagnosis and treatment of these patients each day. Psychological problems in such patients could be detected early and promptly addressed.

In summary, COVID-19 patients have significant anxiety, depression, and sleep disorders, especially in people aged 50 and older. Medical staff should focus on the psychological state of such patients, strengthen health education, and actively conduct psychological counseling. By reducing anxiety and depression through targeted interventions during a psychological crisis, it is possible to reduce the psychological harm caused by the epidemic and subsequent psychosocial problems.

\section{References}

[1]. Huang, C., et al., Clinical features of patients infected with 2019 novel coronavirus in Wuhan, China. Lancet, 2020(2020V395N10223:497-506).

[2]. World, H.O., WHO Director-General's remarks at the media briefing on 2019-nCoV on 11 February 2020. 2020(February).

[3]. Li, Q., et al., Early Transmission Dynamics in Wuhan, China, of Novel Coronavirus-Infected Pneumonia. N Engl J Med, 2020(Jan 29).

[4]. Wang, D., et al., Clinical Characteristics of 138 Hospitalized Patients With 2019 Novel CoronavirusInfected Pneumonia in Wuhan, China. JAMA, 2020(Feb 07).

[5]. Lin, M., et al., Epidemiologic and Clinical Characteristics of Novel Coronavirus Infections Involving 13 Patients Outside Wuhan, China. JAMA, 2020(Feb 07).

[6]. Carlos, W., et al., Novel Wuhan (2019-nCoV) Coronavirus. Am J Respir Crit Care Med, 2020. 201 (4): P7-P8 (Feb 15).

[7]. National, H.C.O.C., A notice on the issuance of guidelines for emergency psychological crisis intervention in pneumonia for novel coronavirus infections. http://www.nhc.gov.cn/xcs/zhengcwj/20 2001/6adc08b966594253b2b791be5c3b9467.shtml, 2020(accessed Feb 11, 2020; in Chinese). 
[8]. General Office of the National Health and Health Commission, Diagnosis and Treatment of New Coronavirus Pneumonia (Trial Version 6), 2020.

[9]. Wei Hua and Li Tingyu, The impact of new coronavirus pneumonia on the psychology of different populations and suggestions for psychological intervention. Journal of Pediatric Pharmacy, 2020 (http://kns.cnki.net/kcms/detail/50.1156.r .20200218.1651.001.html).

[10]. Cheng Li, et al., Anxiety status and influencing factors in patients with corona virus disease 2019. Zhejiang Medical Journal, 2020(2020.42.4.2020-357).

[11]. Chen Jinxiu and Chen Baoyi, Psychoanalysis and nursing of 36 cases of suspected atypical pneumonia. Modern Medicine and Health, 2004. 20 (5).

[12]. Kamara, S., et al., Mental health care during the Ebola virus disease outbreak in Sierra Leone. Bull World Health Organ, 2017(Dec 01): p. 95(12):842-847.

[13]. Leung, G., et al., Longitudinal assessment of community psychobehavioral responses during and after the 2003 outbreak of severe acute respiratory syndrome in Hong Kong. Clin Infect Dis, 2005. 40(12):1713-20(Jun 15).

[14]. Toth, L., Sleep, sleep deprivation and infectious disease: studies in animals. Adv Neuroimmunol, 1995(5(1):79-92).

[15]. Krueger, J.M., Infectious disease and sleep: involvement of neuroendocrine-neuroimmune mechanisms. Int J Neurosci, 1990(Apr): p. 51(3-4):359-62.

[16]. Krueger, J.M., Sleep as a host defense: its regulation by microbial products and cytokines. Clin Immunol Immunopathol, 1990(Nov): p. 57(2):188-99.

\section{Declarations}

Ethic approval was obtained by Fifth Affiliated Hospital of Sun Yat-sen University Ethics Committee. We conducted the "Survey of anxiety and depression in patients with suspected and confirmed cases of COVID - 19 during hospitalization and the isolation" research and prepared to publish this paper.

We conducted the "Survey of anxiety and depression in patients with suspected and confirmed cases of COVID - 19 during hospitalization and the isolation" research. Before we started the study, we had obtained the patients' consent and signed the book in person. All participants agreed to use the findings for research and publication.

\section{Figures}




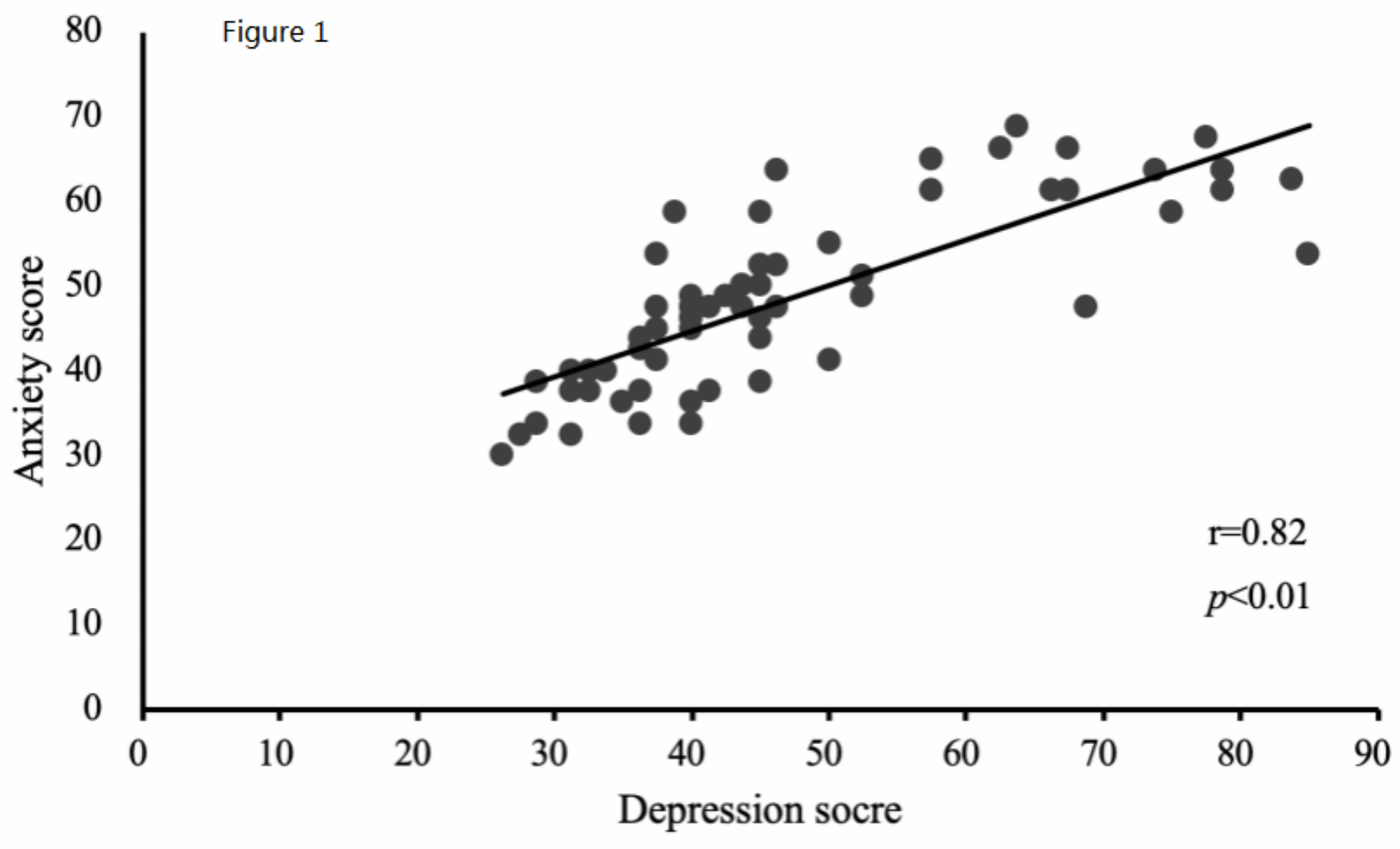

Figure 1

Relationship between the anxiety and depression scores (each point indicates one individual) 


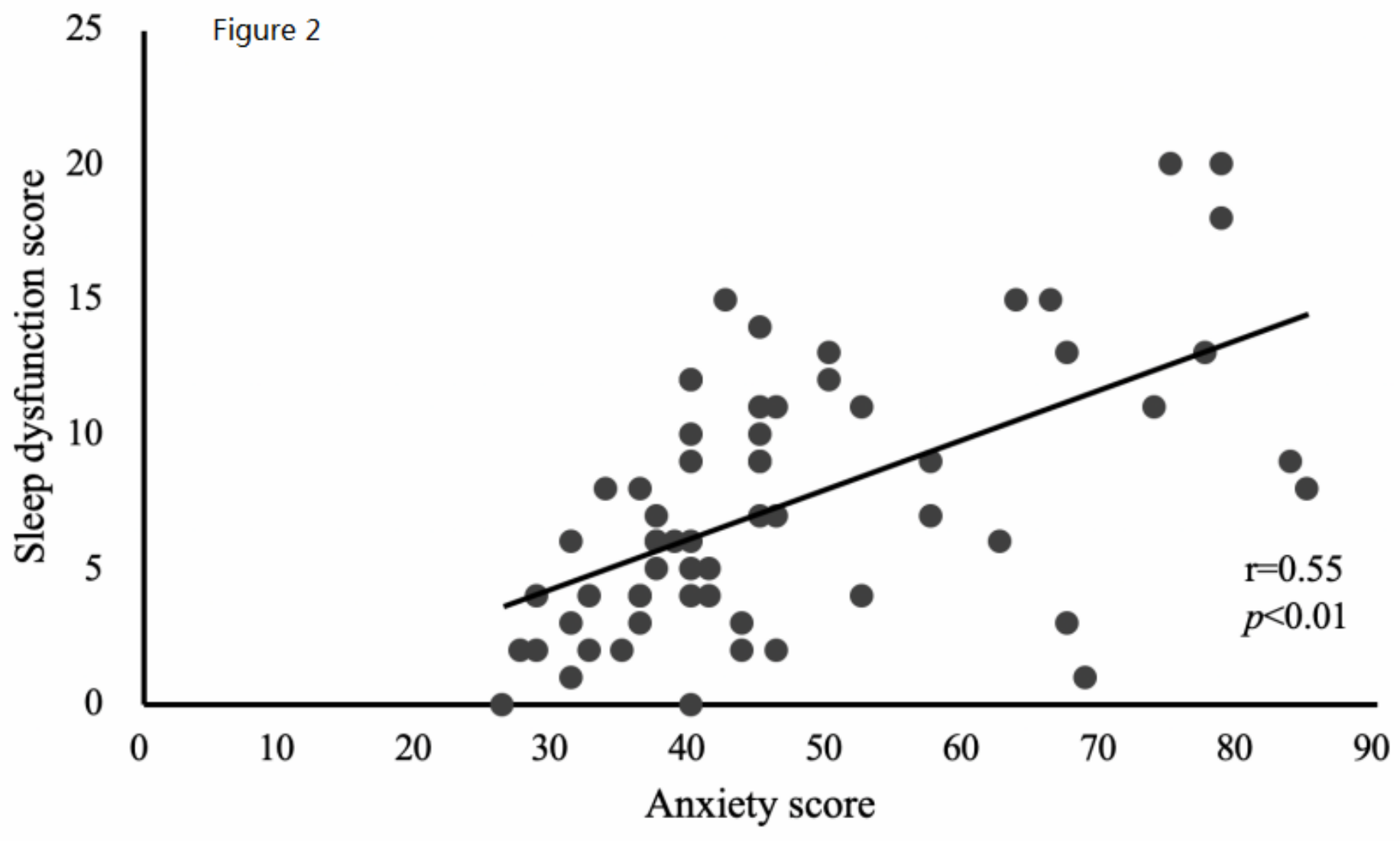

Figure 2

Relationship between the anxiety and sleep dysfunction scores (each point indicates one individual) 


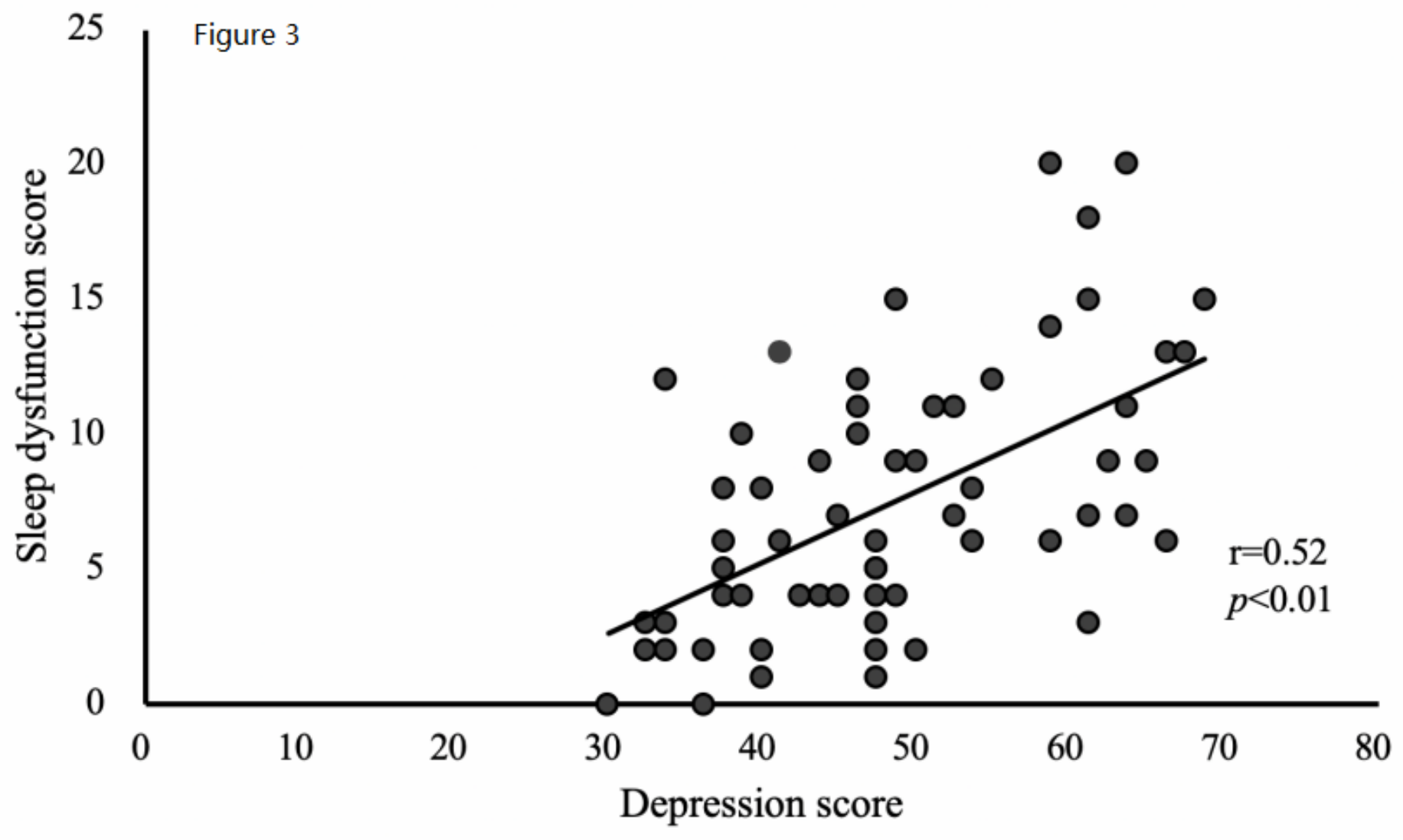

Figure 3

Relationship between the depression and sleep dysfunction scores (each point indicates one individual) 